\title{
Vers la déconstruction d'un apprentissage idéologique : Des "représentations-obstacles" dans l'enseignement des langues en France
}

\section{Gilles Forlot}

\section{OpenEdition}

12 Journals

\section{Édition électronique}

URL : http://journals.openedition.org/rdlc/2063

DOI : $10.4000 /$ rdlc.2063

ISSN : 1958-5772

Éditeur

ACEDLE

\section{Référence électronique}

Gilles Forlot, «Vers la déconstruction d'un apprentissage idéologique : Des "représentationsobstacles" dans l'enseignement des langues en France », Recherches en didactique des langues et des cultures [En ligne], 6-1 | 2009, mis en ligne le 01 avril 2009, consulté le 24 avril 2019. URL : http:// journals.openedition.org/rdlc/2063; DOI : 10.4000/rdlc.2063

\section{Ce document a été généré automatiquement le 24 avril 2019}

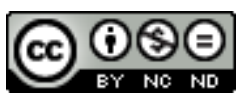

Recherches en didactique des langues et des cultures is licensed under a Creative Commons AttributionNonCommercial-NoDerivatives 4.0 International License 


\title{
Vers la déconstruction d'un apprentissage idéologique : Des "représentations-obstacles" dans l'enseignement des langues en France
}

\author{
Gilles Forlot
}

\section{Introduction et objectifs}

1 En France, l'institution scolaire s'est donné depuis quelque temps des objectifs clairs en termes d'apprentissage des langues à l'école. Dans la lignée du Cadre commun de référence pour les langues publié par le Conseil de l'Europe, la Loi d'orientation et de programme pour l'avenir de l'école d'avril 2005, dans son article 25 , prévoit de manière explicite l'étude d'une langue étrangère à l'école primaire. L'objectif que se donne l'institution, outre celui de préserver et d'encourager la diversité linguistique dans le système éducatif, est d'amener les élèves des écoles de France à une meilleure maîtrise de la langue première et de développer la connaissance de deux langues vivantes à la fin de leurs études secondaires.

2 Cet article ${ }^{1}$ a pour objectif d'exposer les résultats (provisoires et introductifs) d'une enquête, initiée en 2005, dont la problématique relève de ce qui ne semble pas encore être une interrogation centrale de l'institution: le développement et l'évolution des représentations sociolangagières des premiers acteurs (chronologiquement) chargés de l'enseignement des LVE en France, les professeurs des écoles.

3 À l'origine de cette enquête, il s'agissait de faire un état des lieux des pratiques langagières d'étudiants inscrits à la préparation du concours de professeurs des écoles (désormais CRPE) dans la région du Nord/Pas-de-Calais. L'objectif était d'identifier les pratiques quotidiennes ayant trait à l'usage et à la maîtrise d'autres parlers que la "langue 
maternelle" (le français pour une grande majorité d'entre eux) ainsi que de cerner les discours circulant sur ces langues et parlers autres.

4 En gardant à l'esprit que des résultats plutôt positifs sont ressortis des expériences à grande échelle menées en Europe dans le domaine de l'éveil aux langues (Candelier, 2003) et de l'appropriation des techniques d'éveil par les enseignants (Bernaus \& al., 2003), nous avons toutefois constitué l'enquête sur l'hypothèse suivante: en France, les étudiantsprofesseurs et les professeurs débutants n'ont qu'un contact modéré avec l'univers des langues étrangères, une attitude souvent timorée face à l'apprentissage et la pratique de langues étrangères, un positionnement souvent négatif vis-à-vis de l'écart à la norme (exemple de la pratique stigmatisé des patois) et pour finir des représentations négatives (c'est-à-dire simplifiées et stéréotypées) des langues étrangères.

Ce sont ces dernières que nous qualifions de représentations-obstacles, sachant que toute représentation ne ressortit pas forcément au stéréotype négatif, comme l'ont montré nombres de recherche, notamment en psychologie sociale, en sociologie et dans les études littéraires (Amossy \& Herschberg Pierrot, 2005). Le postulat de départ était que ces professeurs en devenir ou débutants, à l'aube d'une carrière dédiée à l'éducation (notamment l'éducation à la diversité), entretiennent des rapports quelque peu difficiles avec ce que l'on pourrait globalement qualifier d'altérité linguistique

6 Aussi, le but de cette recherche est, à terme, multidimensionnel : il est en effet à la fois descriptif (faire un état des lieux), il est analytique et explicatif (tenter d'identifier les causes de la formation de certaines représentations) et pour finir, il a une visée prospective et formative, en ce sens qu'identifier les conséquences de ces représentations sur la pratique du terrain (enseignement/apprentissage) permettra de trouver des modalités et des stratégies de contournement des obstacles.

\section{Méthodologie}

\subsection{Corpus nordiste}

7 Avant de pouvoir agir sur les pratiques de terrain, il convient de se faire une idée de la situation, de cartographier l'espace représentationnel, pour ainsi dire. Ainsi, l'outil de production des données, ici le questionnaire, doit être considéré comme le pendant de l'étude sur le terrain. Ainsi, en 2006, dans l'Académie de Lille, le questionnaire se composait de 30 questions de différentes formes (fermées, à choix multiples ou échelonnées) dont neuf questions mixtes et d'une liste de 9 langues sur lesquelles on demandait aux participants de s'exprimer en quelques lignes.

Comme le questionnaire avait pour objectif de dévoiler des considérations épilinguistiques ${ }^{2}$ et représentationnelles des candidats au CRPE, les sous-ensembles sur lesquels les répondants étaient interrogés concernaient les déterminants sociaux (sexe, âge, lieu d'habitation, scolarité); les pratiques linguistiques (langues parlées, cours de langues suivis, séjours à l'étranger, fréquentation de non-francophones); les pratiques "médiatiques" dans les langues autres que le français (lecture, écoute de la radio, visionnage de la télévision, utilisation de l'internet); la pratique dialectale (attitudes vis-à-vis des parlers picards); le rapport immigration/langue(s); les langues vivantes étrangères dans le système scolaire (en termes qualitatifs et quantitatifs) ; et, pour finir, 
des commentaires libres sur les neuf langues suivantes : l'anglais, l'allemand, l'espagnol, l'arabe, le portugais, le russe, le chinois, le néerlandais et le latin.

En 2005-2006, 275 questionnaires ont été récupérés auprès d'étudiants inscrits au module obligatoire de langue vivante étrangère des centres IUFM de Douai (Nord) et d'Arras (Pasde-Calais), soit environ $70 \%$ des étudiants ciblés. Au total, seuls 29 questionnaires provenaient de répondants masculins, reflet de la large majorité féminine dans les IUFM. Les anglicistes constituaient 166 questionnaires, alors que les hispanistes avaient produit 81 questionnaires et les germanistes 28 questionnaires.

Pour garantir la transférabilité des analyses à la population source de l'enquête (Martin, 2005) et pour ne pas réduire à peau de chagrin les corpus de germanistes et d'hispanistes, ces chiffres ont fait l'objet d'une manipulation statistique afin d'assurer la représentativité de chacune des trois langues "majeures" enseignées dans l'académie de Lille (cf. Forlot 2006 pour de plus amples détails méthodologiques). Nous avons ainsi obtenu la répartition suivante, hommes et femmes mélangés : 174 personnes, dont 107 anglicistes (61,5\% des interrogés), 28 germanistes (16\%) et 39 hispanistes $(22,4 \%)$.

\subsection{Corpus picard}

11 L'enquête a été poursuivie en 2007 dans l'académie d'Amiens, notamment à Beauvais, important centre IUFM chargé de la formation des professeurs stagiaires. 133 enseignants débutants (professeurs des écoles stagiaires, communément appelés PE2) ont été interrogés. Ceci constitue près de $65 \%$ du total des stagiaires inscrits dans ce centre et venant des trois départements de l'académie (Somme, Aisne et Oise). Pour des raisons logistiques inhérentes au fonctionnement du centre IUFM, tous les stagiaires étaient inscrits au module d'anglais au moment de la passation du questionnaire.

12 Le questionnaire consistait en une vingtaine de questions échelonnées, sur le modèle utilisé en psychologie sociale : chaque répondant exprimait, sur une échelle de 5 points, son accord ou son désaccord avec une affirmation donnée sur les thèmes suivants : (1) leur perception de leur niveau de compétences en LVE au collège et au lycée; (2) leur appréciation de la langue anglaise, de la culture anglaise/britannique et de la culture américaine ; (3) leur perception des raisons expliquant la réussite ou le développement de compétences en LVE ; (4) leur perception de l'institutionnalisation des LVE à l'école ${ }^{3}$; (5) leur perception de la facilité ou de la difficulté des LVE et de la proximité entre anglais et français.

13 À l'issue de ces questions aux réponses échelonnées, les professeurs stagiaires - tous anglicistes de facto - étaient invités à livrer leurs commentaires sur les langues suivantes, à peu près comme leurs collègues du Nord-Pas de Calais l'avaient fait un an auparavant : l'anglais, l'allemand, l'espagnol, l'italien, l'arabe, le portugais, le chinois, le russe, le latin et le picard, langue de France (au sens de Cerquiglini, 2003) que partagent, à quelques différences dialectale près, les locuteurs de Picardie et du Nord-Pas-de-Calais (et que ces derniers appellent communément "patois" ou "ch'ti"). 


\section{3. $1^{\text {er }}$ constat : un accès limité à la diversité linguistique}

14 Parmi les obstacles identifiés dans les deux parties de l'étude, et plus particulièrement celle menée dans l'académie de Lille - parce que le questionnaire se prêtait davantage à ce genre d'analyse -, nous avions constaté que les pratiques langagières des candidats au professorat de écoles se révélaient peu ouvertes sur la diversité linguistique (cf. Forlot, 2006 : 125-126). Nous avions mis en lumière trois types de carences que nous pourrions qualifier d'"alterlinguistiques"4: l'absence de pratique linguistique "étrangère" au domicile $^{5}$, des séjours peu nombreux dans des pays étrangers et une exposition très faible aux médias et à l'internet en langues étrangères.

\subsection{Une pratique des langues étrangères peu fréquente}

Le taux très faible de répondants immigrés eux-mêmes ou issus de la migration (8 réponses positives, soit 4,6\% de l'échantillon) est sans doute représentatif de la population candidate au CRPE et laisse supposer que les pratiques linguistiques interpersonnelles déclarées, au foyer, à l'étranger et dans les relations sociales demeurent modérées. Pour vérifier cette hypothèse, nous avons analysé les réponses concernant la pratique d'une autre langue que le français au foyer en affectant à chaque réponse un code représentant l'intensité de chaque pratique déclarée. Ainsi, on obtient une fourchette de "scores" de 0 à 2 , dans laquelle le score 0 équivaut à "aucune pratique". Le score 1 signifie que le répondant parle une LVE à la maison, jusqu'à concurrence de $50 \%$ du temps. Pour finir, le score 2 est attribué aux répondants qui déclarent parler plus de la moitié du temps dans une LVE au domicile.

Le tableau suivant présente les résultats, révélant une moyenne de scores de 0,7 :

\begin{tabular}{|l|c|c|}
\hline Les répondants & $\begin{array}{c}\text { Score } \\
\text { attribué }\end{array}$ & $\begin{array}{c}\text { Nombre et } \\
\text { pourcentage }\end{array}$ \\
\hline Ne parlent pas d'autre langue au foyer & $\mathbf{0}$ & $\mathbf{1 6 2 ( 9 3 , 1 \% )}$ \\
\hline $\begin{array}{l}\text { Parlent une autre langue, de rarement à la moitié du } \\
\text { temps }\end{array}$ & $\mathbf{1}$ & $\mathbf{1 1 ( 6 , 3 \% )}$ \\
\hline Parlent de la moitié du temps à tout le temps & $\mathbf{1}(\mathbf{0 , 5 \% )}$ \\
\hline \multicolumn{2}{|c|}{ Moyenne des scores:0,7 } \\
\hline
\end{tabular}

Tableau 1 - Pratique déclarée d'une langue étrangère au foyer $(\mathrm{n}=174)$.

\subsection{Une faible fréquence du contact "alterlinguistique"}

Dans le même questionnaire, deux questions interrogeaient les participants sur leurs séjours éventuels à l'étranger (voir tableau 2, ci-dessous) et sur leur fréquentation de personnes dont le français n'est pas la langue première. L'objectif de cette partie du 
questionnaire était d'évaluer si des voyages à l'étranger et les contacts directs avec des non francophones sont susceptibles de compenser une pratique individuelle familiale monolingue.

L'hypothèse de départ, celle d'un faible contact, a pu être confirmée, à savoir que les étudiants-professeurs des écoles interrogées sont rarement enclins à interagir avec des non francophones : 34 d'entre eux, soit 9,7\% déclarent avoir interagi au moins une fois par semaine pendant plus de 2 semaines avec un locuteur non francophone.

En ce qui concerne les séjours à l'étranger, le score moyen (entre 0 et 4 ) était de 0,98 , soit légèrement en dessous de la réponse type "un ou plusieurs séjours de 3 jours à 3 semaines". La majorité de ces séjours, d'ailleurs, se révèlent inférieurs à une dizaine de jours et sont, à n'en pas douter, des séjours de vacances (pour de plus amples détails statistiques, voir Forlot 2006). On notera tout de même que les durées de séjour supérieures à 8 semaines étaient deux fois supérieures parmi les germanistes et les hispanistes. Les causes de cet état de fait sont difficiles à circonscrire et sont probablement multiples, de nature sociale et politique, sans lien convaincant avec la langue étrangère pratiquée.

Il est sans doute raisonnable de rester prudent quant aux effets de ces séjours plus ou moins longs ou fréquents et donc de ce contact accentué. En effet, l'hypothèse que le contact intense permet de diminuer la formation des stéréotypes a été infirmée dans certains travaux de psychologie sociale, notamment par les théoriciens du conflit social (Sherif \& Sherif, 1969). D'autres ont même avancé que dans certains cas, le contact pouvait accentuer le stéréotypage (Ladmiral \& Lipiansky, 1989).

\subsection{L'usage des médias comme exposition à la diversité ?}

21 Par l'usage des médias, on entend ici la confrontation à une partie ou tous les moyens de diffusion d'informations extérieures à la vie quotidienne de chaque participant à l'enquête. Il pouvait donc s'agir de journaux, de radio, de télévision ou d'internet, en l'occurrence en langues étrangères. Ici, l'hypothèse n'est pas, comme on vient de le dire, qu'il y a une corrélation entre la fréquence de l'exposition aux médias et la formulation de stéréotypes sociolinguistiques négatifs, mais plutôt que la pratique médiatique est un indicateur d'intérêt pour des pratiques langagières différentes.

De surcroit, comme on sait que l'offre des médias en langues étrangères par les créneaux classiques de la distribution est plutôt limitée, on considérera le choix d'avoir recours à ces médias en langues étrangères soit - au bas mot - comme la quête explicite d'un maintien ou d'un développement de compétences linguistiques, soit comme un positionnement interculturel affirmé.

\begin{tabular}{|c|c|}
\hline Taux moyen de pratique & Total : Nombre et \% du nombre total \\
\hline aucune pratique & $76(43,7 \%)$ \\
\hline Pratique très rare & $74(42,5 \%)$ \\
\hline Pratique rare à mensuelle & $18(10,3 \%)$ \\
\hline
\end{tabular}


Pratique courante à fréquente

De bimensuelle à hebdomadaire

$6(3,4 \%)$

Tableau 2 - Pratiques déclarées d'usage des médias écrits, radiodiffusés, télévisés et en ligne en langues étrangères

La lecture du tableau révèle que l'essentiel des pratiques déclarées d'usage des médias se situent à des taux faibles, de l'usage inexistant à un contact rare d'un ou deux médias. Dans le codage des réponses, les pratiques "rare" et "très rare" correspondaient respectivement aux réponses "une à deux fois par mois" et "rarement". Les étudiantsprofesseurs à l'étude peuvent donc être décrits comme des usagers des moyens de communications francophones (ou doublés, pour ce qui est des émissions télévisées et des œuvres cinématographiques). Cela reflète aussi certainement une tendance, dans leurs pratiques quotidiennes, à considérer comme marginale l'ouverture à une altérité linguistique et de se soumettre à une idéologie linguistique monolingue héritée de diverses traditions centralisatrices et unificatrices françaises. Ces traditions ont participé de la construction de la nation française et des attributs mythologiques qui en constituent le pendant linguistique: le français comme langue nationale (Balibar, 1993; Baggioni, 1997a; 1997b), le monolinguisme et sa construction idéologique (Achard, 1987; Boyer, 2000, 2003 ; Blanchet, 2004).

On sait que cette idéologie a des conséquences notoires sur le positionnement d'une majorité de Français face à la pratique et l'apprentissage des langues étrangères (Dabène, 1994 ; 1997). Il parait certain que ces attitudes ne sont pas sans poser de problème à une époque où un autre discours circule en Europe : celui du plurilinguisme. La question qui se pose alors ici est d'importance, car nous sommes au cœur même du dispositif d'enseignement et d'apprentissage scolaires : les professeurs des écoles, dans leur grande majorité, n'échappent pas au monolinguisme, par manque de compétences - déclarées tout du moins - tout autant que par idéologie subie, alors que l'on constate aussi chez nombre d'entre eux la tendance à accompagner leur pratique linguistique d'un discours pourtant bien actuel insistant sur la nécessité d'apprendre des LVE.

Ce paradoxe s'explique par la tension entre des instructions officielles de plus en plus explicites sur le rôle qu'ils doivent jouer dans l'ouverture des enfants du primaire aux langues et aux cultures des autres et leurs propres positionnements épilinguistiques, forgées tout au long de leur scolarité et reposant en grande partie sur des représentations des langues que l'on pourrait qualifier d'"hyper-normatives". Cette tension, outre qu'elle est sans doute génératrice d'une insécurité linguistique accentuée, crée et entretient aussi une posture contradictoire vis-à-vis des apprentissages langagiers, posture qui ressortit en grande partie à une forme de "schizolinguisme"t.

\section{4. $2^{\mathrm{e}}$ constat : des représentations épilinguistiques en forme d'obstacles}

\subsection{Une perception culturellement et historiquement située}

Des recherches ont déjà montré (Boyer, 2003 ; Auger \& Clerc, 2006, entre autres) que les perceptions des langues renvoyaient souvent à une vision globalisante du peuple (ou du pays) dans ses aspects souvent négatifs. On peut considérer cette perception comme 
ethnoculturelle. Aussi, l'espagnol jouit ici d'un capital de sympathie qui joue certainement en sa faveur tout autant que sa prétendue utilité sur le marché des langues dans le monde. Cette langue est ainsi considérée comme "chaleureuse"7, "enjouée", "joyeuse", "sensuelle". Elle fait fréquemment penser au soleil, aux vacances, au flamenco, à la fête (cf. Angousture, 2004 ; Auger \& Clerc, 2006)... Au contraire, l'allemand est souvent associé à la dureté, à la sévérité ; c'est aux yeux de beaucoup une langue "agressive", "froide", voire "militaire". La sympathie que génère le picard est réelle dans les deux régions étudiées, mais les discours sollicités sont très souvent à l'image de ce que l'idéologie monolingue a construit des parlers régionaux: le picard est "amusant", "rigolo" ; il est "sympathique" mais "campagnard", "rustique", c'est une langue qui "fait péquenot".

Parmi les onze langues soumises aux commentaires des répondants des deux académies, deux font l'objet de peu d'évaluations de nature historique et culturelle: l'anglais et l'arabe. La première est rarement - ou de plus en plus rarement - considérée comme directement rattachée à un peuple, mais plutôt à une pratique langagière quasimondialisée. Dans le second volet de la recherche, en Picardie, seuls 6,7 \% des répondants donnent une réponse de nature ethnoculturelle. La langue arabe, quant à elle, fait l'objet de peu de commentaires ou en tout cas de commentaires prudents, évitant les jugements de nature ethnique, historique ou culturelle.

\subsection{Un stéréotypage épilinguistique persistant}

28 Comme on pouvait s'y attendre, le corpus révèle une série de représentations "classiques", notamment épilinguistiques, concernant diverses langues. La langue y est ici soit perçue en elle-même, dans ses dimensions typologiques et formelles, soit en tant qu'objet d'apprentissage.

\subsubsection{La langue en elle-même : l'aspect formel et la dimension esthétique}

Là aussi, l'espagnol jouit d'une bonne opinion, avec une large majorité d'opinions favorables sur ce que les répondants estiment être sa "beauté", sa "douceur", sa "mélodie", son "rythme" (53 items). L’anglais, lui aussi, est qualifié de "fluide" et de "mélodieux" et on signale souvent sa "musicalité" et sa "souplesse". Sans surprise, les répondants font de fréquentes allusions à la graphie différente de l'arabe et du chinois. On souligne aussi la beauté présumée de ces graphies.

Inversement, deux langues sont décrites comme étant laides, dures et inélégantes, soit intrinsèquement (l'allemand) soit parce qu'on perçoit en elles rien de plus qu'une déformation du français ou un parler peu raffiné : c'est bien sûr le cas des parlers picards, dans les deux régions étudiées. Le picard est souvent - et paradoxalement - considéré comme un attribut de la richesse culturelle de la région et du patrimoine, mais estimé très laid et "fautif" vis-à-vis d'un français normé dont les répondants supposent d'ailleurs souvent qu'il en est un rejeton rural et incorrect. Certains s'étonnent même à l'idée que le picard puisse se trouver dans la liste de langues sur lesquelles on les interroge, comme en témoigne ces trois extraits : "ce n'est pas sérieux", "pourquoi cette question? ", "ce n'est pas une langue ! " (cf. aussi Landrecies, 2001). 


\subsubsection{La langue en tant qu'objet d'apprentissage vécu ou représenté} langues qui véhiculent un capital sur un marché d'échanges de diverses natures. Bien entendu, on pense avant tout à l'anglais. Si les répondants ne livrent que peu de commentaires sur les caractéristiques épilinguistiques ou métalinguistiques de l'anglais, cette langue est en revanche perçue, dans $76 \%$ des questionnaires, comme "indispensable", "incontournable", "essentielle" parce qu"'internationale", "universelle". Certains évoquent même sa domination "écrasante", son "hégémonie" sur le marché des langues, en France ou à travers le monde.

Dans les discours des professeurs stagiaires, deux langues émergent par leur importance de nos jours: l'espagnol et le chinois. Toutefois, cette conception instrumentale de la langue demeure, à l'exception de l'anglais, plutôt modeste. Cela s'explique en partie par le cursus de ces enseignants, marqués à la fois par des objectifs d'enseignement peu axés sur le monde du travail ${ }^{8}$, mais aussi par une assez faible exposition à l'altérité linguistique, comme nous l'avons démontré ci-dessus. L'allemand, on pouvait aussi s'y attendre, fait l'objet de peu de commentaires concernant son utilité, sans doute parce qu'elle ne convainc pas les répondants, mais aussi sûrement parce que l'espace représentationnel de cette langue est occupé par les commentaires épi et métalinguistiques. 


\subsection{La langue en tant qu'outil et véhicule identitaire} conception utilitaire : l'arabe et le picard. Il semble que ces deux langues assument une fonction communicative communautaire : l'arabe, fréquemment perçu ${ }^{9}$ comme la langue que partagent les gens - et plus spécifiquement les élèves et les parents - issus de l'immigration maghrébine - et le picard, considéré comme utile dans le cadre de la communication intracommunautaire ou patrimoniale, par exemple dans l'usage familial ou dans l'utilisation symbolique (et sporadique) pour affirmer son appartenance régionale ou identitaire.

Le picard semble ici assumer ce rôle identitaire de façon assez forte, mais de façon quelque peu paradoxale : à la fois considéré comme un parler laid et souvent incorrect (cf. 4.2.1 ci-dessus) par une majorité d'interrogés, il est aussi l'objet de fréquentes références à la famille, à la survie du patrimoine, à l'histoire régionale. Parce que ce point mérite de plus amples développements et parce que les limites de cet article nous obligent à différer l'analyse approfondie dans des publications ultérieures, nous dirons ceci brièvement : les attitudes sociolangagières des professeurs interrogés dans le Nord-Pas-de-Calais et en Picardie insistent sur la nécessité de préserver l'identité linguistique régionale à travers la pratique du picard, mais elles soulignent aussi les dangers de l'enseignement de cette langue pour l'apprentissage de la langue dite "nationale", le français (voir aussi Dawson, 2004). Ce positionnement est d'autant plus paradoxal que nous avons montré (cf. ci-dessous et Forlot, 2006) que pour ces professeurs, l'école est au cœur des apprentissages linguistiques.

Nous terminerons ce portrait épilinguistique en notant que les références faites à l'allemand comme langue du peuple allemand, notamment en lien avec les première et seconde guerres mondiales ou avec le nazisme, ont presque disparu (4 items sur 307 répondants, soit $1,3 \%$ des interrogés) et qu'une autre caractéristique quasi-identitaire ressort plus fréquemment : celle de la langue scolaire des bons élèves, des bonnes classes. Cette représentation, comme celles liées à la difficulté ou à la facilité supposée de telle ou telle langue, s'expliquent en partie dans le fait que nombre d'interrogés, a fortiori des professeurs stagiaires ou des candidats au concours de l'enseignement, perçoivent les langues comme une prérogative essentiellement scolaire: leurs représentations, renforcés par leurs pratiques alterlinguistiques limitées (cf. ci-dessus), font de l'école le lieu unique où les langues s'acquièrent et, pour la plupart des répondants, se pratiquent.

\section{Quelques pistes pour déconstruire ces représentations-obstacles}

\subsection{Développer une culture sociolinguistique et une conscientisation métalinguistique}

$\mathrm{Au}$ vu de ces résultats quantitatifs et après l'analyse des discours représentationnels, on peut s'interroger sur la culture sociolinguistique et la conscience métalinguistique souvent limitées des professeurs des écoles interrogés. Leur formation universitaire ne semble leur donner accès qu'à une reproduction de schémas normatifs qui ne leur autorisent que peu de prises de distance vis-à-vis des pratiques langagières. Si le discours 
didactique insiste depuis longtemps sur la différence théorique et pratique entre la faute et l'erreur, beaucoup de jeunes enseignants n'assimilent finalement que la nuance théorique et reproduisent des schémas auxquels eux-mêmes ont été soumis dans leur cursus universitaire et, plus encore, dans leur parcours de candidats au CRPE ${ }^{10}$. Pour contribuer à la démonstration, nous proposons quelques exemples de perceptions didactiques encore entretenues par les jeunes enseignants, du primaire comme du secondaire.

\subsubsection{Exemple 1 : Lutter contre une vision approximative de la proximité des langues}

Il s'agit pourtant d'une caractéristique linguistique à faire fructifier en milieu d'apprentissage captif. Le corpus révèle cependant un certain nombre de positionnements représentationnels qui semblent entraver l'accès serein à l'apprentissage des langues. Une majorité des PE2 interrogés, $73 \%$ du corpus picard, perçoivent par exemple l'anglais comme une langue fortement éloignée du français, pour deux raisons: (a) elle est, à plus ou moins juste titre, placée dans leur savoir métalinguistique, dans le groupe des langues germaniques, alors qu'elle est à forte composante lexicale romane (cf. Crépin, 1967; Walter, 2001; Robert, 2008; Forlot \& Beaucamp, 2008) ; (b) le lexique de l'anglais, quand il est représenté comme étant proche de l'anglais, est davantage associé à un danger qu'à un avantage.

En ce sens, la notion de "faux amis" a pris le pas sur les avantages liés à la proximité lexicale (notamment la transparence écrite) et, largement surestimée (Klein, 2007), elle s'est installée dans l'imaginaire des apprenants - dont nos répondants anglicistes comme étant la caractéristique essentielle du lexique des langues étrangères. Ce réflexe n'est pas propre aux anglicistes. Il provient de processus d'apprentissage linguistiques dans lesquels ont longtemps prévalu (et nous gageons que c'est encore le cas) la hantise que la première langue joue un rôle d'interférence négative et génère ainsi des séries d'erreurs interlinguistiques (Dabène, 1994), les fameux "faux-amis". Aussi, la langue s'apprend-elle en cloisonnement total des autres, comme si l'élève-apprenant de L2 était vierge linguistiquement (Forlot \& Beaucamp, 2008).

\subsubsection{Exemple 2 : Dépasser la vision lexicaliste et "hyper-grammaticale" de la langue}

Ceci paraîtra tantôt évident aux tenants de la méthode communicative, tantôt scandaleux aux partisans du retour à un apprentissage prétendument structurée et lexicalement enrichissant de la langue (Bentolila, 2007). Mais il ne s'agit pas de dire ici que l'on doit ou que l'on peut - apprendre une langue sans apprendre son lexique et sans construire des phrases avec sa syntaxe. Il s'agit plutôt de contrecarrer une conception "classique" de la langue étrangère où la méthode d'enseignement, subie par ces PE1 et PE2 eux-mêmes jusqu'à l'université, reposait pour l'essentiel sur de l'apprentissage par cœur - donc décontextualisé - de règles et de listes de mots lexicaux ou grammaticaux. En guise d'exemple, on citera les nombreux commentaires des répondants au sujet de la longueur quasi-rédhibitoire des mots de l'allemand, alors qu'une conceptualisation métalinguistique saine - à offrir aussi aux non germanistes - leur aurait enseigné qu'il ne s'agit là que de compositions lexicales et d'un artifice de la transcription graphique de cette langue. 
43 La grammaire, bien sûr, n'est pas en reste : quand on interroge les PE2 sur la grammaire de l'anglais, les réponses portent essentiellement sur la question du present perfect, alors que ceux sur l'allemand portent sur les déclinaisons. Cette sur-focalisation sur les formes perçues comme difficiles, reproduite par des générations d'enseignants de langue, ont contribué à leur tour à créer des effets d'insécurité linguistique (la peur de la "faute") et renforcé une vision hyper-normative de futurs enseignants du primaire reflétant encore l'idéologie monolingue dont nous parlions plus haut.

44 Ceci explique sans doute que de nombreux professeurs du primaire, face aux défis que constitue l'enseignement des langues étrangères à l'école, raisonnent surtout en termes de difficultés, comme s'il s'agissait de la caractéristique essentielle de l'apprentissage linguistique. Dans le corpus picard par exemple, $86 \%$ des enseignants interrogés estiment qu'il existe, dans l'absolu, des langues plus difficiles que d'autres. Forgés dans la culture de la norme et de la difficulté de s'y conformer, il est donc logique que 83,5\% d'entre eux considèrent le français comme une langue difficile à apprendre pour un étranger.

\subsection{Agir par étapes pour corriger les représentations-obstacles}

La formation des enseignants du primaire - et à moyen terme de ceux du secondaire gagnerait à reposer sur quelques étapes visant à forger des représentations renouvelées des langues et de l'apprentissage linguistique. Nous en proposons quatre.

\subsubsection{La prise de conscience de l'existence des représentations}

On ne contestera pas que les représentations sont bel et bien nécessaires dans la structuration du réel et la construction de l'identité (Amossy \& Herberg Pierrot, 2005). On soulignera toutefois que les formations linguistiques des enseignants du primaire (et du secondaire) consistent souvent à identifier les représentations des élèves ${ }^{11}$, alors que les enseignants eux-mêmes, comme nous l'avons montré, ont des conceptions épilinguistiques qui nous paraissent faire obstacle. Une piste à exploiter serait la propre conscience, en discours, que ces enseignants ont de leurs stéréotypes sociolinguistiques. Voici deux exemples qui pourraient servir de points de départ à une déconstruction des représentations-obstacles :

a) "l'italien, j'ai trop de clichés sur cette langue" (PE2, corpus picard).

b) "on parle portugais en rajoutant des 'y' et des 'ch', genre 'yest chui portugaich'... mais ça doit pas être vraiment ça" (PE1, corpus nordiste).

\subsubsection{L'identification de la nature de ces représentations}

À partir de ce travail d'identification, il serait envisageable de construire avec les enseignants des typologies de stéréotypes leur permettant de prendre de la distance visà-vis de ceux-ci. Par exemple, on peut recenser les domaines stéréotypiques suivants (cf. Forlot, 2006) :

50 a) la langue et ses supposées vertus intrinsèques (l'espagnol et l'italien = mélodieux ; l'allemand = guttural... cf. Yaguello, 2004);

51 b) la langue comme reflet plus ou moins positif de la culture (par ex., l'espagnol = le flamenco ; l'italien = la pizza... cf. section 3.1. ci-dessus) ; 
à profit ce qui parait étrange à l'apprenant en vue précisément de le mémoriser. Par exemple, on peut montrer, dans une sensibilisation au plurilinguisme, que les sons qui paraissent difficiles aux apprenants francophones, comme le redouté <th> dévoisé de l'anglais $(/ \theta /)$, se retrouve aussi en espagnol (d'Espagne), ou encore que certains sons que les Français perçoivent comme désagréables à l'oreille ne sont pas cantonnés à certaines langues: le /x/ de l'allemand (par ex. : achtung, lachen), qui contribue sans doute à la vision de dureté que projette cette langue, se retrouve aussi fréquemment dans bien d'autres langues, comme l'arabe, le néerlandais, les langues celtiques, ainsi que dans une des langues que les interrogés classent précisément parmi les plus mélodieuses et euphoniques : l'espagnol. (par ex. : Juan, lejos... ).

\section{Pour conclure, quelques orientations didactiques à l'usage du "législateur éducatif"...}

Nous avons vu que les premiers acteurs chargés de l'enseignement des langues en France, les professeurs des écoles, étaient encore fortement sous l'influence de représentations qui ne paraissent pas faciliter ni garantir le développement d'un plurilinguisme en France. Or, depuis quelques années, les directives pédagogiques officielles insistent sur le développement de cette "philosophie", explicitement ou implicitement en relation avec la mondialisation croissante et le développement d'une Europe unie. Toutefois, malgré les efforts des chercheurs et pédagogues dans le domaine, le plurilinguisme ne peut naître de lui-même. Au contraire, il se crée avant tout dans l'imaginaire sociolangagier collectif.

De ce fait, si les programmes d'éveil aux langues misent sur l'apprentissage de la diversité linguistique par les enfants (Candelier, 2003; Kervran, 2006, entre autres), il semble essentiel d'initier les enseignants à des programmes du même type, sous peine de voir se reproduire et perdurer des positionnements stéréotypiques vis-à-vis des diverses langues de l'espace scolaire. En d'autres termes, la première priorité de l'école est sans doute la reconnaissance de sa propre hétérogénéité linguistique (Castellotti, 2008), et une formation adéquate des jeunes enseignants, avant que ceux-ci ne soient eux-mêmes 
chargés de former à un éveil au plurilinguisme dont ils ne sont sans doute pas tous convaincus.

En second lieu, il faut se donner les moyens de ses ambitions: l'institution scolaire appelle à s'ouvrir au plurilinguisme et à développer l'interaction orale, tout en donnant finalement peu de marge de manœuvre à ceux qui veulent faire communiquer en langue étrangère. En témoignent depuis des années les épreuves du baccalauréat et les cursus universitaires - en dehors des filières linguistiques proprement dites - pour l'essentiel axé sur la compréhension et la production écrite (analyse textuelle, traduction, littérature...).

En dernier lieu, comment ne pas appeler, à tous les niveaux, à désabsolutiser la langue (Hambye \& al., 2007), à objectiver celle-ci, à s'affranchir d'une vision académicienne et fétichiste de celle-ci (Klinkenberg, 2001), obsessionnellement normative et génératrice d'insécurité linguistique et de stéréotypages dépréciatifs? "Trop de régionalismes dans les performances des candidats...", indiquait un rapport de jury du concours de recrutement des professeurs des écoles en 2005 dans l'académie de Lille. Si la tolérance à l'altérité et à la diversité est une des clés de voûte de la construction harmonieuse de notre société de demain, le travail sur les représentations sociolinguistiques ne pourra qu'y contribuer positivement.

\section{BIBLIOGRAPHIE}

Achard, P. (1987). "Un idéal monolingue". In Vermès, G. \& Boutet, J. (dir.). France pays multilingue, vol. 1. Paris : L'Harmattan. pp. 38-57.

Amossy, R. \& Herberg Pierrot, A. (2005). Stéréotypes et clichés. Paris : Armand Colin.

Angousture, A. (2004). Espagne, idées reçues. Paris : Le Cavalier Blanc.

Auger, N. (2003). "Les représentations interculturelles dans des manuels de français langue étrangère en usage dans l'Union Européenne". In Alén Garabato, C., Auger, N., Gardies, P. \& Kotul, E. Les représentations interculturelles en didactique des langues-cultures. Enquêtes et analyses. Paris :

l'Harmattan. pp. 35-71.

Auger, N. \& Clerc S. (2006). "Les représentations de l'hispanité chez des élèves français : constat et urgence d'une pédagogie interculturelle". In La cultura del otro : español en Francia, francés en España - La culture de l'autre : espagnol en France, français en Espagne. http:// www.culturadelotro.us.es/actasehfi/pdf/5auger_cerc.pdf (consulté le 10/12/2007). pp. 980-992.

Baggioni, D. (1997a). "Langue nationale". In Moreau M.-L. (dir.). In Sociolinguistique. Les concepts de base. Sprimont : Mardaga. pp. 189-192.

Baggioni, D. (1997b). Langues et nations en Europe. Paris : Payot.

Balibar, R. (1993). Le colinguisme. Paris : PUF.

Bentolila, A. (2007). Le verbe contre la barbarie. Apprendre à nos enfants à vivre ensemble. Paris : Odile Jacob. 
Blanchet, Ph. (2004). "Enseigner les langues de France ?". Cahiers Pédagogiques nº 123. http:// www.cahiers-pedagogiques.com/.

Bernaus M., Kervran M. \& Noguerol A. (2003). "L'appropriation de l'approche par les enseignants". In Candelier, M. (dir.). Janua Linguarum - la porte des langues. L'introduction de l'éveil aux langues dans le curriculum. Strasbourg : Éditions du Conseil de l'Europe. pp. 139-170.

Billiez, J. \& Dabène L. (1984). Recherches sur la situation sociolinguistique des jeunes issus de l'immigration. Grenoble : Université Stendhal-Centre de didactique des langues.

Boyer, H. (2000). "Ni concurrence, ni déviance : l'unilinguisme français dans ses œuvres". Lengas 48. pp. 89-101.

Boyer, H. (2003). De l'autre côté du discours. Recherches sur les représentations communautaires. Paris : L'Harmattan.

Candelier, M. (dir.) (2003). Janua Linguarum - la porte des langues. L'introduction de l'éveil aux langues dans le curriculum. Strasbourg : Éditions du Conseil de l'Europe.

Castellotti, V. (2008). "L'école française et les langues des enfants : quelle mobilisation de parcours plurilingues et pluriculturels ?". In Chiss, J.-L. (dir.). Immigration, école et didactique du français. Paris : Didier. pp. 231-279.

Cerquiglini, B. (dir.) (2003). Les langues de France. Paris : PUF.

Crépin, A. (1967). Histoire de la langue anglaise. Paris : PUF.

Dabène, L. (1994). Repères sociolinguistiques pour l'enseignement des langues : les situations plurilingues. Paris : Hachette.

Dabène, L. (1997). "L'image des langues et leur apprentissage". In Matthey, M. (dir.). Les langues et leurs images. Neuchâtel : IRDP. pp. 19-23.

Dawson, A. (2004). "'L'patois s'apprind tout seu' : les pièges de l'enseignement du picard". Études de Linguistique Appliquée no 136. pp. 487-498.

Forlot, G. (2006). "Des pratiques aux stéréotypes sociolinguistiques d'étudiants-professeurs. Résultats préliminaires d'une enquête et pistes de recherche". Spirale no 38. pp. 123-140.

Forlot G. \& Beaucamp J. (2008). "Heurs et malheurs de la proximité linguistique dans l'enseignement de l'anglais au primaire". Études de Linguistique Appliquée nº 149. pp. 77-92.

Hambye, Ph., Delcourt I., Forlot G. \& Lucchini S. (2007). Francophones et plurilingues. Le rapport à la langue française et au plurilinguisme des Belges issus de l'immigration. Rapport de recherche au Ministère de la culture de la Communauté française Wallonie-Bruxelles, Université catholique de Louvain : Louvain-la-Neuve.

Kervran, M. (2006). " Pourquoi et comment faire appel à la diversité des langues du monde à l'école primaire". Spirale no 38. pp. 27-35.

Klein, H.G. (2004). "L'eurocompréhension (EuroCom), une méthode de compréhension des langues voisines". Études de Linguistique Appliquée no 136. pp. 403-418.

Klein, H.G. (2007). "0ù en sont les recherches sur l'eurocompréhension ?". Site internet Eurocomresearch. http://eurocomresearch.net/lit/Klein\%20FR.htm (consulté le 15/02/2008)

Klein, H.G. \& Stegmann, T. (2000). EuroComRom - Die sieben Siebe: Romanische Sprachen sofort lesen können. Aachen: Ed. EuroCom vol. 1.

Klinkenberg, J.-M. (2001). La langue et le citoyen. Pour une autre politique de la langue française. Paris : PUF. 
Kotul E. (2003). "Les représentations interculturelles dans les manuels de FLE conçus en Pologne entre 1962 et 1996". In Alén Garabato, C., Auger, N., Gardies, P. \& Kotul, E. Les représentations interculturelles en didactique des langues-cultures. Enquêtes et analyses. Paris : l'Harmattan.

pp. 115-141.

Ladmiral, J.-R. \& Lipiansky E.-M. (1989). La communication interculturelle. Paris, Armand Colin.

Landrecies, J. (2001). "'c'est laid mais ça fait me rire' : Les représentations de l'accent du nord dans une population de stagiaires de l'IUFM de Lille". In Hintze, M.-A. Pooley, T. \& Judge, A. (dir). French Accents: Phonological and Sociolinguistic Perspectives. Londres : AFLS-CILS. pp. 196-217.

Martin, O. (2005). L'enquête et ses méthodes : L'analyse de données quantitatives. Paris : Armand Colin.

Matthey, M. (dir.) (1997). Les langues et leurs images. Neuchâtel : IRDP.

Moore, D. (dir.) (2001). Les représentations des langues et de leur apprentissage. Paris : Didier.

Robert, J.-M. (2004). "Proximité linguistique et pédagogie des langues non maternelles". Études de Linguistique Appliquée no 136. pp 499-511.

Robert, J.-M. (2008). "L’anglais comme langue proche du français ?". Études de Linguistique Appliquée no 149 . pp. 9-20.

Robillard D. (de) (2008). Perspectives alterlinguistiques. Paris: L'Harmattan.

Sherif, M. \& Sherif C. W. (1969). Social Psychology. New York: Harper-Inter.

UNESCO (1995). Stéréotypes culturels et apprentissages des langues. Paris : Commission française pour l'UNESCO.

Walter, H. (2001). Honni soit qui mal y pense. Paris : Laffont.

Yaguello, Marina (2004). Catalogue des idées reçues sur la langue. Paris : Seuil.

\section{NOTES}

1. Cet article est une version remaniée et élargie d'une communication faite au colloque de l'ACEDLE de janvier 2008 à l'Université Marc Bloch de Strasbourg. Nous remercions l'IUFM de l'Académie d'Amiens pour le financement alloué pour la participation à ce colloque

2. Dans cet article, nous employons l'adjectif "épilinguistique" pour faire référence aux positionnements explicites vis-à-vis des langues, en termes de statuts et selon des critères esthétiques, par exemple, ou encore par rapport à des discours s'inscrivant dans une vision essentialiste associant langues et conduites ethno-identitaires. L'adjectif "métalinguistique" fait en revanche ici référence aux positionnements et aux discours sur la langue elle-même, dans ses aspects formels et structurels (par ex. les déclinaisons, temps et aspects, l'orthographe, etc.).

3. Son nouveau statut de discipline obligatoire à l'école, la tendance au tout anglais, la question de son enseignement à la maternelle, l'apprentissage des langues de l'immigration...

4. Cet adjectif s'entend ici comme "marqué par l'expérience du contact à l'altérité et à la diversité linguistique". Pour une approche complète de ce concept, voir Robillard, 2008.

5. Les guillemets visent à rappeler ici qu'en fait, beaucoup de répondants ont au moins une pratique patoisante en compréhension, mais ne la considèrent que rarement comme étrangère. Cf. Forlot 2006 pour un développement sur le sujet.

6. En avril 2008, lors d'une intervention au séminaire de recherche de l'équipe DYNADIV de l'Université François-Rabelais de Tours, nous avons proposé de définir "le schizolinguisme à la française" comme une caractéristique épilinguistique de(s) pratiques et représentations contradictoires d'acteurs sociaux conscients de l'existence d'un bilinguisme ou d'un 
plurilinguisme - quelles qu'en soient l'intensité et la forme - dans l'espace les environnant. Ces acteurs sociaux ont une conduite schizolingue quand ils sont incapables de transgresser la vision monolingue de l'espace social que leur histoire et leur éducation a construite.

7. Toutes les expressions entre guillemets visant à décrire les langues sont des citations extraites des discours des répondants.

8. Position logiquement légitimée par les textes officiels du primaire, qui misent sur l'ouverture culturelle plutôt que sur la capitalisation de ces compétences linguistiques sur un marché du travail lointain pour ces enfants..

9. De façon sans doute un peu simplifiée, à la fois parce que cette conception ne tient pas compte des importantes différences dialectales entre les variantes de l'arabe, même au Maghreb, mais aussi parce que certains jeunes issus de l'immigration maghrébine ne parlent pas l'arabe (cf. Billiez \& Dabène, 1984 ; Hambye \& al., 2007).

10. Il suffit, pour s'en convaincre, de voir les modalités de l'épreuve de langue vivante, consistant ni plus ni moins en la reproduction d'une évaluation de lycée ou d'université, détachée de tout contexte scolaire et/ou didactique. Le moins que l'on puisse dire, c'est que ce type d'épreuve n'apporte que peu de compétences didactiques, métalinguistiques et sociolangagières aux futurs professeurs des écoles.

11. Voir l'abondante littérature existant à ce sujet désormais, par exemple UNESCO, 1995 ; Matthey, 1997 ; Moore, 2001.

12. Par exemple dans les manuels utilisés (cf. Auger, 2003 et Kotul 2003), ou encore les thématiques abordées en classe. Les sentiments d'un snobisme anglais et d'une austérité allemande évoqués plus haut sont, aux dires de professeurs stagiaires rencontrés à l'issue de cette enquête, issus de thématiques redondantes dans leurs scolarités, telles que les aristocratiques public schools britanniques ou la sévérité du régime est-allemand.

\section{RÉSUMÉS}

Cet article s'appuie sur une recherche de terrain en cours dans les IUFM du Nord-Pas de Calais et de Picardie auprès d'environ 300 étudiants au professorat des écoles et professeurs des écoles stagiaires. En combinant une dimension quantitative de nature statistique (par questionnaires) et une approche qualitative d'analyse de discours sollicités, nous exposerons les premières conclusions de l'étude et explorerons les multiples décalages existant entre les objectifs institutionnels dans le domaine des LVE en France et les représentations sociolinguistiques des personnels chargés de les enseigner dans le primaire. On y exposera notamment les difficultés ou parfois les réticences - ressenties par les professeurs des écoles débutants à adhérer à une démarche plurilingue de l'enseignement des LVE qui rompt avec une approche "classique", c'està-dire souvent lexicaliste et grammaticale de l'enseignement. On constate qu'en dépit d'une progressive ouverture aux langues et à la diversité, les positionnements des futurs enseignants $\mathrm{du}$ primaire reflètent encore une idéologie monolingue et des postures à tendance "schizolingue", acquise dès l'enfance et tout au long de la scolarité et reposant en grande partie sur des représentations des langues que l'on pourrait qualifier d'"hyper-normatives" et génératrice d'insécurité linguistique. On s'interrogera pour finir sur le rôle de la formation des enseignants dans la construction, la reproduction - et éventuellement la déconstruction - de ces représentations. 
This article is based on research carried out in several teaching colleges of Northern France and Picardy, where approximately 300 students and teacher trainees were given questionnaires aimed at identifying their sociolinguistic construals. Combining quantitative and qualitative data, the article presents our initial conclusions and explores the various discrepancies between the official objectives of the French educational authorities regarding language teaching and the sociolinguistic stereotypes of those in charge of teaching languages in primary school. We will show the difficulties and sometimes the reluctance of teachers to adhere to the current multilingual orientations for language teaching in France because these orientations challenge the classical approach they themselves went through in their own learning of foreign languages (e.g. vocabulary and grammar based memorisation, drills, etc.). As these sociolinguistic attitudes contribute to linguistic insecurity and the maintenance of monolingual and at times "schizolingual" ideologies in France, we will try to illustrate how teacher training can be helpful in the construction (or the deconstruction) of such construals.

\section{INDEX}

Mots-clés : représentations sociolinguistiques, enseignement des langues, école primaire, formation des enseignants

Keywords : sociolinguistic construals, language teaching, primary education, teacher training

\section{AUTEUR}

\section{GILLES FORLOT}

Gilles Forlot est depuis 2007 maître de conférences à l'Université de Picardie Jules-Verne (IUFMUPJV) et membre du Laboratoire d'études sociolinguistiques sur les contacts de langues et la politique linguistique (LESCLAP, JE 2466). Il a été chercheur à l'Ontario Institute for Studies in Education de l'Université de Toronto de 1993 à 1997 puis à l'Université catholique de Louvain de 2002 à 2007.

Courriel : gilles.forlot[at]u-picardie.fr

Toile : http://www.u-picardie.fr/LESCLaP

Adresse : Centre IUFM/UPJV, 3 rue Bossuet, 60000 Beauvais, France. 